\title{
A Solution for Goal-oriented Policy Refinement in NFV Management and Orchestration Systems
}

\author{
Michel Bonfim $^{1,2}$, Fred Freitas ${ }^{1}$, Stênio Fernandes ${ }^{1}$ \\ ${ }^{1}$ Centro de Informática - Universidade Federal de Pernambuco (UFPE), \\ Recife - Pernambuco - Brasil - Caixa Postal 50740-560 \\ ${ }^{2}$ Universidade Federal do Ceará (UFC) - Campus Quixadá \\ Quixadá - Ceará - Brasil - Caixa Postal 63902-580 \\ \{msb6, fred,sflf\}@cin.ufpe.br
}

\begin{abstract}
In this thesis, we propose an automated policy refinement procedure for NFV-MANO systems based on planning and description logic reasoning. It is implemented in ATOM, a framework that enables NFV-MANO to record high-level goals and performs a fully automated policy refinement that derives enforceable rules to govern NFV-MANO behavioral choices. Besides, ATOM provides a semantic verification system to find inconsistencies among policies. Experiments have shown that our solution scales well considering realistic scenarios with different sizes.
\end{abstract}

\section{Motivation and Problem Statement}

Network Function Virtualization (NFV) technology will play a significant role in 5G networks since they allow network programmability and the fast delivery of new services. However, management in NFV scenarios is a complex task once delivering such Virtual Network Functions (VNFs) and Network Services (NSs) over the physical infrastructure requires flexible and adaptable NFV Management and Orchestration (NFV-MANO) systems [Giotis et al. 2015]. The increasing complexity of the NFV-MANO has widened the gap between human intention and the managed system behavior. Therefore, new solutions are certainly necessary to reduce this gap and improve these complex systems' management.

In this context, Policy-based Management (PBM) systems can be used to enforce NFV-MANO functions as a way to deal with this increased complexity. PBM systems are the key enablers to provide flexibility and adaptability in NFV-MANO systems. Assisted by policies, NFV-MANO functions can be provided in an automated fashion, aiming to meet the dynamic requirements of Network Service Orchestration (NSO) and Resource Orchestration (RO). An NFV-PBM system refers to the management of rules governing the behavior of NFV-MANO. However, PBM is not a straightforward task [Riekstin et al. 2016]. This situation is exacerbated when considering its application in the management of NFV systems, since NFV-MANO must offer several functionalities in a flexible and adaptable way. In this scenario, a fundamental issue regarding NFV-PBM systems arises, namely, policy refinement.

Policy Refinement is the process of transforming high-level policies (goals), which are not directly executable in a management system, into directly enforceable, low-level policies [Bandara et al. 2004]. Policy refinement is a nontrivial process, and it remains 
a much-neglected research area. It has been severely dismissed due to its inherent complexity [Machado et al. 2017].

In most systems, policy refinement is done manually [Rochaeli 2009]. Such situation imposes a number of problems. First, whenever the refinement process is required, the presence of an expert is usually necessary. Moreover, refining policies manually for large and complex systems consists of a tedious and challenging task. The scale of changes can be vast, leading to improper specifications of enforceable policies due to human errors. Therefore, for NFV systems, we argue that it is crucial to accomplish this procedure in an automated way. An automated refinement process, based on expert knowledge, should reduce the gap between human intention and the NFV system behavior.

A fully automated refinement process still constitutes an open issue and a highly desired goal. Besides, to the best of our knowledge, no other work in the literature has described an automated refinement scenario applied to NFV-MANO operations. In this thesis, we tackle the issue of providing a functional solution for automated policy refinement in policy-based NFV management and orchestration systems based on planning and Description Logic reasoning.

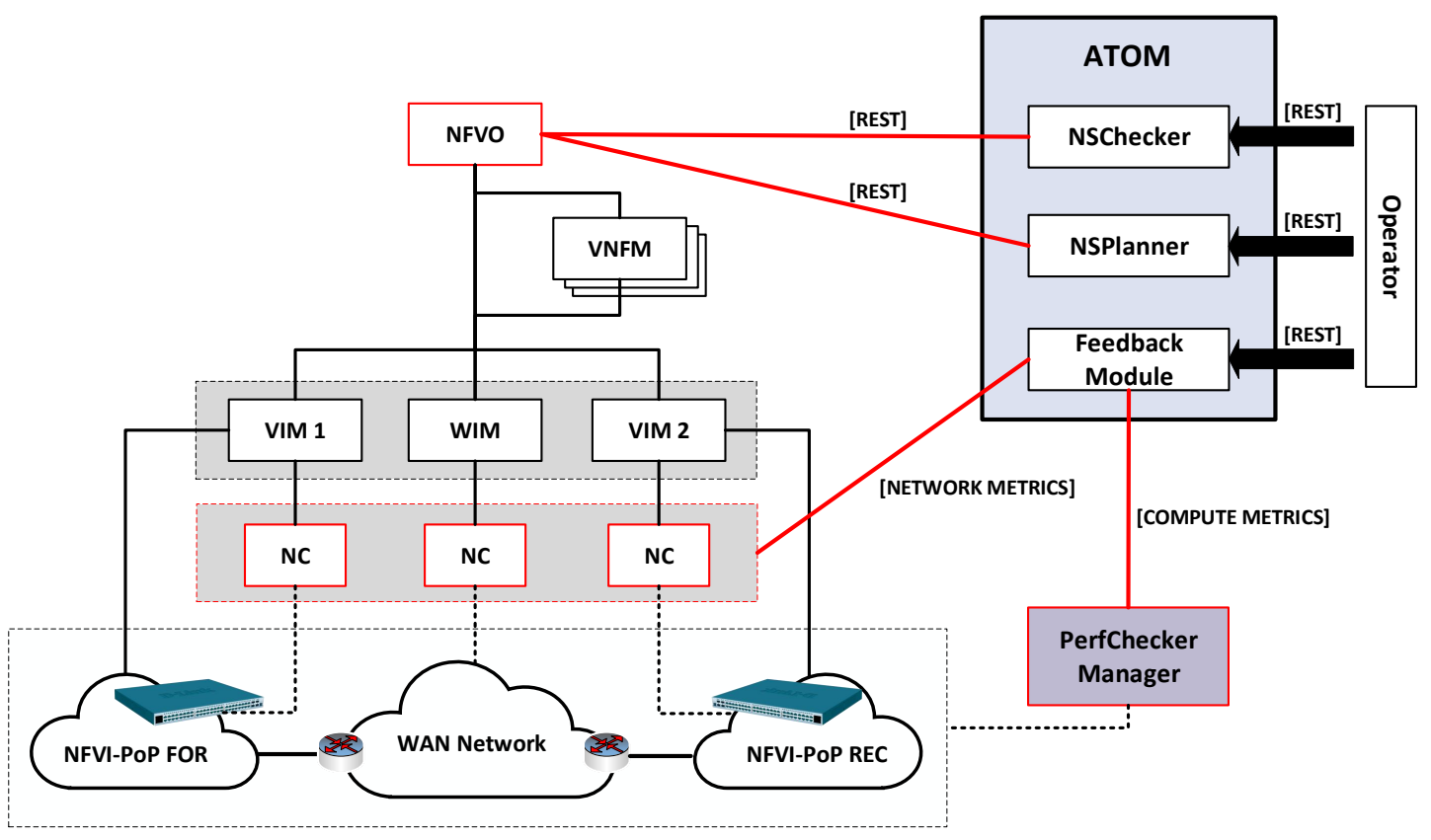

Figure 1. High-level ATOM Architecture.

\section{Proposal}

We propose the AuTomated POlicy Refinement SysteM for NFV (ATOM). It aims to deploy a solution for automated goal-oriented policy refinement for NFV-PBM systems, supporting the following refinement requirements:

- Requirement 1 (RT1): Support to Goal-oriented refinement;

- Requirement 2 (RT2): Support to detection and resolution of policy conflicts (policy analysis); 
- Requirement 3 (RT3): Verification of impact of administrative guidelines on the managed systems' performance;

- Requirement 4 (RT4): Support of runtime decision making in a policy-managed environment;

- Requirement 5 (RT5): Dynamicity regarding current or future system states, and topology changes;

- Requirement 6 (RT6): Capabilities' orchestration.

It is noteworthy that the implementation of an NFV-PBM is not part of the scope of this thesis. Instead, ATOM aims to assist and improve the operation of these solutions. An example of an NFV-PBM architecture can be found in [ETSI 2017].

Figure 1 illustrates a high-level view of the ATOM architecture and how each functional block interacts with the NFV-MANO component. It comprises three (3) independent subsystems: NSChecker, NSPlanner, and Feedback Module. They interact with both NFV-MANO and NFVI to execute their functionality. We highlight them below.

1. NSChecker: is a semantic verification system to find inconsistencies among policies defined in NS Request (NS-Req) (NS Policy (NS-Pol)) and global policies previously created in the NFVI (NFVI Policy (NFVI-Pol)), i.e, applicationspecific policies. NSChecker has three primary functions. First, it enables the operator or NFV-MANO to record both NFVI physical and virtual resources, as well as their policies (NFVI-Pols), working as an NFVI Resources Repository (NFVI-RR). Besides, it helps NFV-MANO to record all instantiated NSs, working as an NFV Instances Repository (NFV-IR). Finally, once an NS-Req is received, NSChecker assists NFV-MANO to record policies extracted from Network Service Descriptor (NSD) and carry out the detection and diagnosis of conflicts between NS-Pols and NFVI-Pols. To achieve its objectives, NSChecker uses a semantic model (ontology) in Web Ontology Language (OWL) 2 [Krötzsch et al. 2012], called Onto-NFV, to describe the NFV Infrastructure (NFVI), NSs, and associated policies. Policy analysis is carried out through Description Logic (DL) inconsistency verification summoned by a DL reasoner;

2. NSPlanner: provides a goal-oriented policy refinement and semantic verification system. It accomplishes three primary functions. First, it enables the operator to record different types of alarms to be used in the refinement process. Second, NSPlanner supports NFV-MANO to record high-level goals extracted from NSD and to perform a fully automated policy refinement, that derives enforceable policies (Event-Condition-Action (ECA) rules) to govern NFV-MANO behavioral choices, while satisfying the goals. Finally, NSPlanner carries out policy analysis between management-specific policies, i.e., rules that will be generated by the refinement process and stored in NFV-PBM to govern system behavior. To attain the above functionalities, NSPlanner combines the efficiency of Hierarchical Task Network (HTN) planning systems with the DL expressivity. Concerning HTN, it relies on a well-founded HTN planner to execute Goal-oriented policy refinement procedures. All the planning domains (e.g., methods, operators, precondition and effects) should be described using this planner's formalism. Besides, NSPlanner proposes the use of an ontology in OWL 2 [Krötzsch et al. 2012], called OntoPlanner. With Onto-Planner at hand, NSPlanner explores the richer DL expressivity to describe in detail state constraints and axioms, facts about the current 
state of the world, and non-functional parameters about actions (e.g., subject, target, trigger events) and maintain them. Besides, policy analysis is carried out through DL inconsistency verification;

3. Feedback Module: a monitoring system that aims to capture monitoring data from both the network and computer resources. Thus, the operator can measure and analyze results and verify if the enforceable policies are fulfilling high-level goals. If any inconsistency is detected, the operator can update the inconsistencies, creating new alarms, for instance.

Jointly, NSChecker and NSPlanner meet the following refinement requirements: RT1, RT2, RT4, RT5, and RT6. To achieve Requirement 3, ATOM relies on the Feedback Module.

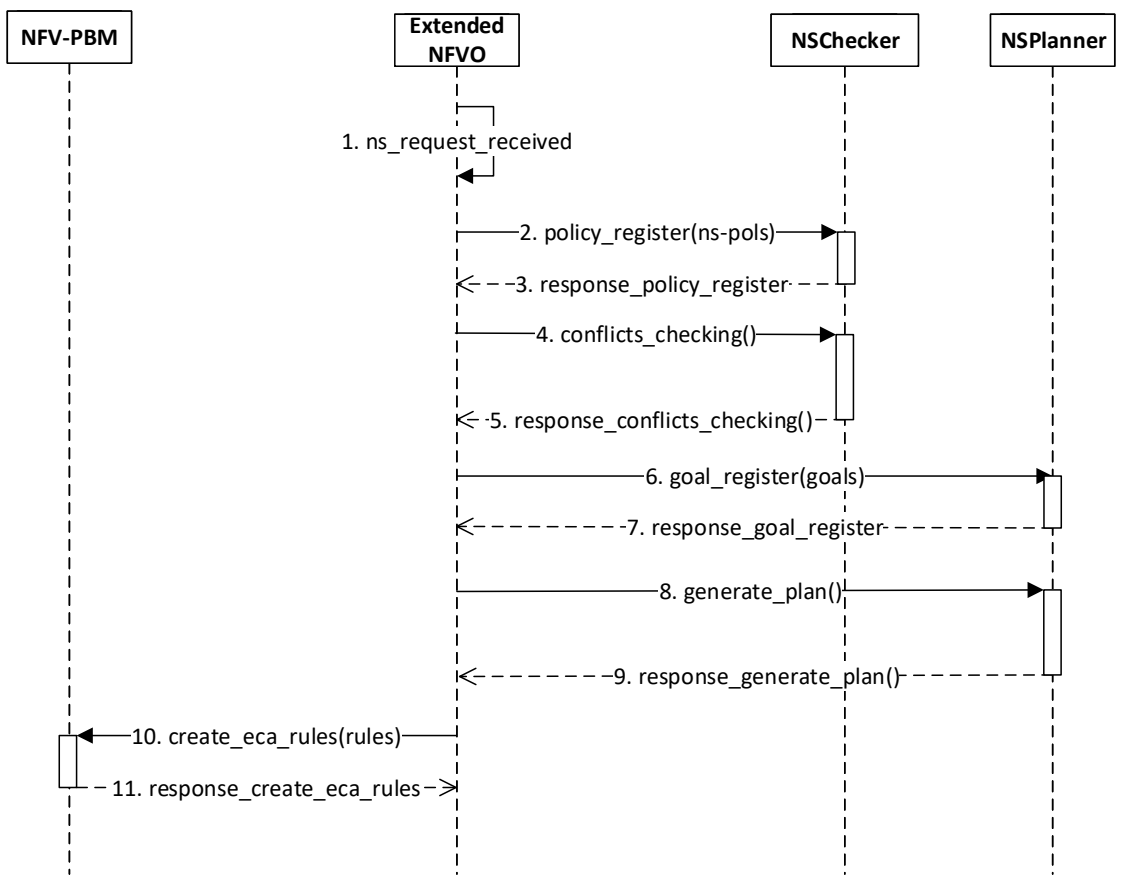

Figure 2. ATOM overview.

Figure 2 displays an overview of ATOM operation, once received an NS-Req. Before starting, we assume that the operator has previously defined all physical and virtual NFVI resources as well as NFVI-Pols using NSChecker. We also assume that all alarms, as well as the planning domain model, have already been created by an expert knowledge using NSPlanner. Hence, having received an NS-Req, the NFV Orchestrator (NFVO) extracts the NS-Pols and requests NSChecker to record those policies (steps 2 and 3). Next, NFVO queries NSChecker for possible conflicts between NS-Pols and NFVI-Pol (steps 4 and 5). In the presence of these conflicts, NSChecker generates possible explanations, and NFVO interrupts its operations to create an alert for whom requested the service. Otherwise, if no conflict is detected, the NFVO proceeds by extracting the goals specified in NS-Req and requiring NSPlanner to record those policies (steps 6 e 7). In its turn, NSPlanner enables NFVO to ask for a plan (sequence of actions) to be performed in order 
to achieve the recorded goals (steps 8 and 9). With the generated plan at hand, NFVO can then request the NFV-PBM to apply the generated ECA rules (steps 10 and 11), enabling automatic control of NFV-MANO functions.

Finally, it is worth noting that the three ATOM subsystems are independent of each other. For example, an operator could deploy them in different Docker ${ }^{1}$ containers.

\section{Results}

We developed an NSChecker prototype in Java, which provides a RESTful interface that can be used by any NFV-MANO available, such as Open Baton ${ }^{2}$ and Open Source $\mathrm{MANO}^{3}$. We validate NSChecker's capability on a small scenario with three typical use cases, showing that it supports conflict detection concerning the following policies: precedence of network functions, resource usage, and location. Finally, we conducted a performance evaluation of our tool over some real topologies. The results demonstrate that NSChecker is efficient even in scenarios with 50,000 NFV Infrastructure Nodes (NFVI-Nodes).

Moreover, we developed an NSPlanner prototype in Java, which provides a RESTful interface that can be used by any NFV-MANO available. We validated NSPlanner's capability under three use cases, showing that it supports conflict detection concerning managing-specific policies. Finally, we conducted a performance evaluation of our tool. The results show that NSPlanner is efficient even in scenarios with 1000 goals and 1000 alarms pre-registered.

Therefore, based on the NSChecker and NSPlanner experiments, we can conclude that ATOM can be properly applied to production environments as an auxiliary tool to an NFV-MANO framework, accomplishing a goal-oriented policy refinement procedure and detecting and diagnosing possible conflicts among policies before the deployment of new NSs.

\section{Related Work}

In this section, we refer only to studies that have relied on logical formalisms in their policy refinement procedures. Table 1 lists differences between these works and ATOM, which is the primary element of our thesis proposal. These differences take into account the requirements presented in Section 2.

It is remarkable that all above works use refinement methods that are known to be undecidable, i.e., there are no guarantees that they can derive an answer [Hölldobler and Kuske 2000]. On the other hand, ATOM uses both DL and HTN in the refinement process, which are methods known to be decidable [Erol et al. 1994, Georgievski and Aiello 2015]. Furthermore, none of the above works met requirements 3 and 6. Only the work of Bandara et al. offered a solution that accomplishes both refinement and policy analysis. In this context, a fully automated refinement process is still an open issue. To the best of our knowledge, no other work in the literature has provided an automated refinement scenario applied to NFV-MANO operations. In this thesis, we

\footnotetext{
${ }^{1}$ Docker website: https://www.docker.com

${ }^{2}$ Open Baton website: https://openbaton.github.io/

${ }^{3}$ Open Source MANO website: https://osm.etsi.org/
} 
Table 1. ATOM - Comparing related works.

\begin{tabular}{|c|c|c|c|c|c|c|c|}
\hline Reference & $\begin{array}{l}\text { Policy Refinement } \\
\text { Method }\end{array}$ & Goal-oriented Refinement (RT1) & Policy Analysis (RT2) & Verification (RT3) & \begin{tabular}{l|} 
Runtime Decision \\
Making (RT4)
\end{tabular} & Dynamicity (RT5) & $\begin{array}{l}\text { Orchestration } \\
\text { Capabilities (RT6) }\end{array}$ \\
\hline $\begin{array}{l}\text { Bandara et al. } \\
\text { (2003) }\end{array}$ & $\begin{array}{l}\text { Event Calculus with } \\
\text { Abductive Reasoning }\end{array}$ & No & No & No & No & No & No \\
\hline $\begin{array}{l}\text { Bandara et al. } \\
(2004,2006)\end{array}$ & $\begin{array}{l}\text { Event Calculus with } \\
\text { Abductive Reasoning }\end{array}$ & \multirow{3}{*}{$\begin{array}{l}\text { Yes } \\
\text { Yes }\end{array}$} & Yes & No & No & No & No \\
\hline $\begin{array}{l}\text { Rubio-Loyola et al. } \\
(2005,2006,2007)\end{array}$ & $\begin{array}{l}\text { Linear Temporal Logic } \\
\text { with Model Checking }\end{array}$ & & No & No & Yes & Yes & No \\
\hline Rochaeli, Taufiq (2009) & $\begin{array}{l}\text { Description Logic-based } \\
\text { Model Checking }\end{array}$ & & No & No & Yes & No & No \\
\hline $\begin{array}{l}\text { Craven et al. } \\
(2010,2011)\end{array}$ & $\begin{array}{l}\text { Event Calculus with } \\
\text { Abductive Reasoning }\end{array}$ & No & No & No & No & Yes & No \\
\hline $\begin{array}{l}\text { Machado et al }(2014, \\
2015,2017)\end{array}$ & $\begin{array}{l}\text { Event Calculus with } \\
\text { Abductive Reasoning }\end{array}$ & \multirow{2}{*}{$\begin{array}{l}\text { Yes } \\
\text { Yes }\end{array}$} & No & No & Yes & Yes & No \\
\hline АТOM & $\begin{array}{l}\text { HTN Planning with } \\
\text { Description Logic }\end{array}$ & & Yes & Yes & Yes & Yes & Yes \\
\hline
\end{tabular}

intend to deal with this problem. As shown in Table 1, ATOM aims to meet all the refinement requirements of an NFV-PBM system.

\section{Contributions and Publications}

In a broader perspective, ATOM achieves the following contributions:

- The Onto-NFV ontology, which deploys a vocabulary with its complex relations and constraints of the domain, expressive enough to describe NFVI, NSs, and associated policies; Onto-NFV supports the description of the following applicationspecific policies: network function precedence, location constraints, and resource usage;

- The Onto-NFV axioms and Semantic Web Rule Language (SWRL) rules that provides a policy analysis of application-specific policies, based on DL reasoning;

- The NSChecker prototype, a semantic verification system that provides to an operator or NFV-MANO an NFVI-RR, an NFV-IR, and a tool to detect and diagnose conflicts between NS-Pols and NFVI-Pols;

- A language for describing goals in NFV environments;

- The Onto-Planner ontology, which offers a vocabulary with its complex relations and constraints of the domain, expressive enough to describe and maintain state constraints and axioms, facts about the current state of the world, and nonfunctional parameters about actions (e.g., subject, target, trigger events);

- Its axioms and SWRL rules analyses management-specific policies, by DL reasoning;

- The NSPlanner prototype, that provides to an operator or NFV-MANO an automatic goal-oriented policy refinement procedure. Besides, it relies on a semantic verification system to detect and diagnose conflicts between management-specific policies;

- The modeling of a planning domain for the fault management problem in NFV systems;

- The Feedback Module that enables the operator to verify if the enforceable policies are fulfilling the high-level goals. If any inconsistency is detected, the operator is able to eliminate this inconsistency by updating the refinement pattern.

The ATOM source code is available at the following GitHub repositories: https://github.com/michelsb/atom and https://github.com/ michelsb/atom-feedback.

So far, we have published the following papers related to the thesis: 
1. BONFIM, M. S.; SOUZA, R. R. ; COUTINHO, E. F. ; DIAS, K. L. ; FERNANDES, S. F. L. . Identifying Performance Bottlenecks in Software Data Planes for Cloud-based NFV Services. In: IEEE/IFIP Network Operations and Management Symposium, 2018, Taipei. Proceedings of the 2018 IEEE NOMS [Bonfim et al. 2018]. (Qualis: A2)

2. BONFIM, M. S.; DIAS, K. L. ; FERNANDES, S. F. L. . Integrated NFV/SDN Architectures: A Systematic Literature Review. ACM Computing Surveys, 2019. [Bonfim et al. 2019c] (Qualis: A1)

3. BONFIM, M. S.; FREITAS F. ; FERNANDES, S. F. L. . A Semantic-based Policy Analysis Solution for the Deployment of NFV Services. IEEE Transactions on Network and Service Management (TNSM), 2019 [Bonfim et al. 2019b]. (Qualis: B1)

4. BONFIM, M.; DIAS, K.; FERNANDES, S. . SFCMon: An Efficient and Scalable Monitoring System for Network Flows in SFC-enabled Domains. In: PRE-IETF WORKSHOP (WPIETF), 6. , 2019, Belém. Proceedings of the VI Pre-IETF Workshop. Porto Alegre: Sociedade Brasileira de Computação, July 2019. ISSN 2595-6388 [Bonfim et al. 2019a]. (Sem Qualis, Best Paper Award)

Additionally, there are three publications indirectly related to the thesis:

1. BARBOSA, R. R. ; REGO, P. A. L. ; BONFIM, M. S. ; CALlADO, A. C*. Análise de Desempenho das Tecnologias de Virtualização de Rede da Plataforma OpenStack. In: Workshop em Desempenho de Sistemas Computacionais e de Comunicação (WPerformance), 2018, Natal. Porto Alegre: Sociedade Brasileira de Computação, 2018. DOI: https://doi.org/10. 5753/wperformance.2018.3334. (Qualis: B3)

2. AZEVEDO D., BONFIM, M, LIMA L. and FERNANDES S., "Towards an Accurate Bandwidth Estimation Tool for 802.11n Wireless Networks," 2018 IEEE Symposium on Computers and Communications (ISCC), Natal, 2018, pp. 0048600491. DOI: 10.1109/ISCC.2018.8538751 [Azevedo et al. 2018]. (Qualis: A2)

3. BONFIM, M.; SANTOS, M.; DIAS, K.; FERNANDES, S.. A Real-Time Attack Defense Framework for 5G Network Slicing. Wiley Software, Practice \& Experience, 2019 [Bonfim et al. 2020]. (Qualis: A2)

\section{References}

Azevedo, D., Bonfim, M., Lima, L., and Fernandes, S. (2018). Towards an accurate bandwidth estimation tool for 802.11n wireless networks. In 2018 IEEE Symposium on Computers and Communications (ISCC), pages 00486-00491.

Bandara, A. K., Lupu, E., Moffett, J. D., and Russo, A. (2004). A goal-based approach to policy refinement. In 5th IEEE International Workshop on Policies for Distributed Systems and Networks (POLICY 2004), 7-9 June 2004, Yorktown Heights, NY, USA, pages 229-239.

Bonfim, M., Dias, K., and Fernandes, S. (2019a). Sfcmon: An efficient and scalable monitoring system for network flows in sfc-enabled domains. In Proceedings of the VI Pre-IETF Workshop, Porto Alegre, RS, Brasil. SBC. 
Bonfim, M., Freitas, F., and Fernandes, S. (2019b). A semantic-based policy analysis solution for the deployment of nfv services. IEEE Transactions on Network and Service Management, 16(3):1005-1018.

Bonfim, M., Roque, R., Coutinho, E., Dias, K., and Fernandes, S. (2018). Identifying performance bottlenecks in software data planes for cloud-based nfv services. In NOMS 2018 - 2018 IEEE/IFIP Network Operations and Management Symposium, pages 1-7.

Bonfim, M., Santos, M., Dias, K., and Fernandes, S. (2020). A real-time attack defense framework for 5g network slicing. Software: Practice and Experience, 50(7):12281257.

Bonfim, M. S., Dias, K. L., and Fernandes, S. F. L. (2019c). Integrated nfv/sdn architectures: A systematic literature review. ACM Comput. Surv., 51(6):114:1-114:39.

Erol, K., Hendler, J., and Nau, D. S. (1994). Htn planning: Complexity and expressivity. In Proceedings of the Twelfth National Conference on Artificial Intelligence (Vol. 2), AAAI'94, pages 1123-1128, Menlo Park, CA, USA. American Association for Artificial Intelligence.

ETSI (2017). Network functions virtualisation - management and orchestration - report on policy management in mano (release 3). ETSI GR NFV-IFA 023 V3.1.1.

Georgievski, I. and Aiello, M. (2015). Htn planning: Overview, comparison, and beyond. Artificial Intelligence, 222:124 - 156.

Giotis, K., Kryftis, Y., and Maglaris, V. (2015). Policy-based Orchestration of NFV Services in Software-Defined Networks. IEEE Network Softwarization (NetSoft), 605243(605243).

Hölldobler, S. and Kuske, D. (2000). The boundary between decidable and undecidable fragments of the fluent calculus. In Parigot, M. and Voronkov, A., editors, Logic for Programming and Automated Reasoning, pages 436-450, Berlin, Heidelberg. Springer Berlin Heidelberg.

Krötzsch, M., Patel-Schneider, P., Rudolph, S., Hitzler, P., and Parsia, B. (2012). OWL 2 web ontology language primer (second edition). Technical report, W3C. http://www.w3.org/TR/2012/REC-owl2-primer-20121211/.

Machado, C. C., Wickboldt, J. A., Granville, L. Z., and Filho, A. E. S. (2017). ARKHAM: an advanced refinement toolkit for handling service level agreements in softwaredefined networking. J. Network and Computer Applications, 90:1-16.

Riekstin, A. C., Januario, G. C., Rodrigues, B. B., Nascimento, V. T., de Brito Carvalho, T. C. M., and Meirosu, C. (2016). A survey of policy refinement methods as a support for sustainable networks. IEEE Communications Surveys and Tutorials, 18(1):222-235.

Rochaeli, T. (2009). An Automated Policy Refinement Process Supported by Expert Knowledge. PhD thesis, Technische Universitat, Darmstadt. 